\title{
COVID-19 e saúde mental:
}

\section{potencias impactos e estratégias}

\section{de atenção psicossocial}

\author{
Daiane Borges Machado \\ Camila Silveira Silva Teixeira ${ }^{2}$ \\ Aline dos Santos Rocha ${ }^{3}$ \\ Flávia Jôse Oliveira Alves ${ }^{4}$
}

1 Psicóloga e mestre pela Universidade Federal da Bahia (UFBA), doutora em Epidemiologia e Saúde das populações pela London School of Hygiene and Tropical Medicine. Pesquisadora na Harvard Medical School, Rede CoVida e do Centro de Integração de Dados e Conhecimentos para Saúde (Cidacs) da Fundação Oswaldo Cruz (Fiocruz).

2 Nutricionista, mestra em Saúde Pública pela Universidade Federal do Ceará (UFC). Doutoranda em Saúde Pública pelo Instituto de Saúde Coletiva (ISC) da Universidade Federal da Bahia (UFBA) e pesquisadora da Rede CoVida e Centro de Integração de Dados e Conhecimentos para Saúde (Cidacs) da Fundação Oswaldo Cruz (Fiocruz).

3 Nutricionista, mestra e doutoranda em Alimentos, Nutrição e Saúde, Escola de Nutrição da Universidade Federal da Bahia (ENUFBA). Pesquisadora da Rede CoVida e do Centro de Integração de Dados e Conhecimentos para Saúde (Cidacs) da Fundação Oswaldo Cruz (Fiocruz).

4 Psicóloga, mestra em Saúde Comunitária e doutoranda em Saúde Pública pelo Instituto de Saúde Coletiva (ISC) da Universidade Federal da Bahia (UFBA). Pesquisadora da Rede CoVida e do Centro de Integração de Dados e Conhecimentos para Saúde (Cidacs) da Fundação Oswaldo Cruz (Fiocruz). 


\section{Introdução}

Transtornos mentais têm sido relacionados à reativação de doenças como a tuberculose, associados a maiores incidências de doenças cardiovasculares e tende a aumentar em tempos de epidemias. (BAO et al., 2020) No entanto, ainda não está claro como a disseminação catastrófica da pandemia da COVID-19 tem influenciado a saúde mental da população brasileira.

Investigar a saúde mental durante uma epidemia é importante e necessário, pois o comportamento das pessoas pode ajudar a aumentar ou diminuir a propagação de epidemias. Informações atuais têm mostrado que regiões no Brasil, onde o distanciamento físico está sendo atendido, o aumento no número de casos da COVID-19 tem ocorrido de forma desacelerada. (BOLETIM COVIDA, 2020) No entanto, saúde mental é uma disciplina ampla que envolve transtornos mentais, questões psicológicas, comportamentais, socioeconômicas e até aspectos culturais e de acessibilidade. (WHO, 2003) Dessa forma, a saúde mental não é a ausência ou presença de transtornos mentais. A Organização Mundial de Saúde Mental define saúde mental como um estado de bem-estar no qual o indivíduo é capaz de usar e reconhecer suas 
habilidades, lidar com as tensões cotidianas da vida e contribuir para as suas comunidades. (WHO, 2003)

Não se pretendeu no presente capítulo abordar todos os fatores relacionados à saúde mental e nem todos os grupos passíveis de sofrimento psíquico durante a pandemia da COVID-19, o que seria inviável em apenas um documento. Assim como, não se pretendeu aprofundar no tema da violência em tempos de pandemia já que isso envolveria diversos atores e desfechos com classificações distintas conforme a Classificação Internacional de Doenças (CID-10) (ORGANIZAÇÃO MUNDIAL DA SAÚDE, 1994), necessitando, portanto, de uma nova abordagem de revisão de literatura.

O objetivo do presente capítulo foi avaliar as possíveis influências da pandemia da COVID-19 e das estratégias de Distanciamento físico na saúde mental da população brasileira e a manutenção dos serviços de atenção à saúde mental a partir da síntese de conhecimentos científicos. Também se pretendeu fornecer recomendações para subsidiar a tomada de decisão em relação à atenção à saúde de pessoas com transtornos mentais no Brasil durante a pandemia.

\section{Como a pandemia da COVID-19 tem influenciado a saúde mental}

Epidemias podem provocar mal-estar e pensamentos recorrentes sobre possíveis infecções. (HUREMOVIĆ, 2019) Já a preocupação exagerada com a própria saúde e o excesso de vigilância corporal, em situações em que o sujeito se concentra excessivamente em sinais do corpo, como palpitações, frequência respiratória e assim por diante, podem ocasionar sofrimento psíquico. Segundo a teoria cognitiva comportamental - uma das teorias psicológicas -, crenças 
disfuncionais sobre a própria saúde podem levar a interpretações equivocadas das sensações corporais. (BECK, 2013) Essas crenças podem persistir e evoluir para sintomas mais intensos, como, por exemplo, ansiedade e ataques de pânico. (ASMUNDSON et al., 2010) Tais fatores afetam ainda mais os indivíduos que apresentam algum transtorno mental prévio à pandemia, mas não se limita a eles.

O estresse causado pelo iminente risco de ser infectado pelo novo coronavírus e de morte pela doença - especialmente entre as pessoas dos grupos de maior risco -, bem como o estresse causado pela incerteza do impacto econômico, podem aumentar o risco de ansiedade e depressão na população. Estima-se que esses desafios perdurem por pelo menos dois meses, seguidos por vários meses de algum tipo de distanciamento físico.

As informações enganosas (fake news) de agências de notícias e mídias sociais também podem resultar em mais ansiedade e sensação de instabilidade. Atualmente, sabe-se que a COVID-19 se espalha a uma taxa exponencial (BAO et al., 2020), trazendo a sensação de constante risco de infecção, o que pode levar a um estado de hipervigilância corporal. $\mathrm{O}$ estado hipervigilante estendido, por sua vez, pode potencialmente ativar o eixo hipotálamo-hipófise-adrenal e causar níveis elevados de glicocorticoides, que, está relacionado à ansiedade e ao transtorno depressivo. (LUPIEN et al., 2009)

\section{Impacto da mídia na saúde mental}

Os sentimentos mais comuns durante epidemias são o de incerteza, medo e angústia e todos eles podem aumentar o risco de transtornos mentais, como ansiedade e depressão. Durante uma crise, assim como tem sido observado na pandemia da COVID-19, as pessoas procuram informações relacionadas ao evento para se 
manterem informadas. No entanto, quando as informações em canais oficiais estão ausentes ou são obtidas de forma irregular, as pessoas podem ser expostas a informações enganosas, o que pode aumentar os sentimentos negativos. (TORALES et al., 2020)

Embora o uso de dados de mídia social possa fornecer compreensão oportuna do impacto das emergências de saúde pública durante o período epidêmico(LI, S. et al., 2020), a mídia também pode trazer consequências negativas para a saúde mental das pessoas frequentemente expostas às mídias sociais. (GAO et al., 2020) Alto risco de ansiedade e da combinação ansiedade e depressão, foram observadas entre usuários frequentemente expostos às mídias sociais. (GAO et al., 2020) Também foi observado maior nível de ansiedade entre as pessoas que mais acompanharam as notícias relacionadas ao coronavírus. (MOGHANIBASHI-MANSOURIEH, 2020)

É importante o combate à “infodemia” - sensação de aflição e pânico que se instala na população devido à quantidade de informações na mídia -, monitorando e filtrando informações falsas e promovendo informações precisas por meio de colaborações transversais. (GAO et al., 2020) Artigos publicados durante crises epidêmicas anteriores destacaram a importância de liberar atualizações oficiais substanciais em intervalos regulares durante um evento de crise e monitorar as mídias sociais para reduzir a exposição a informações e angústias enganosas. (PURGATO et al., 2018) O público deve prestar atenção às informações que a mídia e a propaganda apresentam, evitando absorver informações enganosas que, inevitavelmente, pode levar ao pânico e à depressão. (WANG, Y. et al., 2020) Recomenda-se também a divulgação de serviços de saúde mental por canais variados, incluindo linha direta, consulta on-line, curso on-line e consulta ambulatorial, com especial atenção aos sinais de depressão e ansiedade. (GAO et al., 2020) Em síntese, a mídia deve relatar objetivamente o progresso da epidemia e aumentar a publicidade de aconselhamento psicológico 
(TIAN et al., 2020), inclusive alertando para a possibilidade de consultas on-line, que podem tornar-se intervenções de porta da entrada para pacientes. (ROY et al., 2020)

No Brasil, o futuro imprevisível da pandemia da COVID-19 também tem sido exacerbado por informações enganosas e desinformação transmitidas por mensagens via WhatsApp e outras mídias sociais, que geram pânico. Na realidade brasileira, a população enfrentou ainda um dilema de recomendações, protagonizado por várias vertentes de orientações, de um lado, as que advêm do presidente da República, de outro, dos estados, municípios ou de organizações internacionais. $\mathrm{O}$ apelo do presidente pelo restabelecimento de escolas e comércio contrariam as recomendações de distanciamento físico. Essas contradições entre os discursos do presidente e demais autoridades estaduais, municipais e da Organização Mundial de Saúde (OMS) criaram um clima de insegurança sanitária que pode provocar efeitos negativos na saúde mental da população, com a antecipação de preocupações e incerteza do prognóstico da pandemia.

\section{Distanciamento físico e saúde mental no Brasil}

Outra preocupação é o imperativo de distanciamento físico, por vezes denominado equivocadamente de quarentena ou isolamento social. O distanciamento social envolve medidas que têm como objetivo reduzir as interações em uma comunidade, como o fechamento de escolas e locais de trabalho, suspensão de alguns tipos de comércio e o cancelamento de eventos. (AQUINO et al., 2020) Entretanto, um termo mais adequado poderia ser "distanciamento físico", visto que as pessoas podem e devem manter contato com a sua rede de apoio - amigos, familiares etc. - por outras vias, como chamadas telefônicas e vídeo, para reduzir o efeito psicológico do 
distanciamento. Huremovio (2019) explica que quarentena significa "separar aqueles que ainda estão saudáveis, mas possivelmente expostos a um agente infeccioso, daqueles que são saudáveis e não foram expostos", enquanto o "isolamento social é um estado de falta de contato entre um indivíduo e a sociedade".

A medida de distanciamento físico é fundamental para evitar a sequência de infecção entre indivíduos infectados da população não afetada. (HUREMOVIĆ, 2019) No entanto, para ser eficaz, essa medida deve ser seguida pela população e complementada com políticas que possibilitem as pessoas a ficarem em casa, incluindo apoio socioeconômico, proteção contra a violência doméstica e atenção em saúde mental para aqueles que precisam, sobretudo no Brasil, onde as pessoas vivenciam os mais diversos contextos sociais e econômicos.

\section{COVID-19 e sofrimento mental em grupos específicos}

O avanço da pandemia aumenta a probabilidade de sofrimento mental e morbidades psiquiátricas em diferentes subpopulações, relacionada às medidas de distanciamento físico, às notícias negativas e ao crescente número de casos confirmados e suspeitos. (LI, W. et al., 2020) Portanto, focar em grupos com maior risco de sofrer problemas de saúde mental durante a pandemia pode ser uma alternativa em situações de limitados recursos.

Médicos, enfermeiros e demais profissionais de saúde podem enfrentar traumas durante o tratamento dos pacientes, especialmente por lidarem com uma nova doença. De acordo com Bao e demais autores (2020), o estresse que eles experimentam pode desencadear transtornos mentais comuns, incluindo Transtorno de Estresse Pós-Traumático (TEPT), ansiedade e transtornos 
depressivos, que por sua vez podem resultar em ameaças que excedem as consequências da própria epidemia da COVID-19.

Os idosos, que estão entre os mais afetados pela pandemia da COVID-19, tanto em termos de gravidade quanto de mortalidade, também apresentam maior chance de sofrerem impactos psicológicos durante este período. (QIU et al., 2020) Estes podem ter maior dificuldade para acessar os serviços de saúde durante a epidemia da COVID-19, pelos desafios no deslocamento e limitado acesso a facilidades tecnológicas - dispositivos e internet quando os atendimentos são realizados on-line. (YANG et al., 2020) Além disso, idosos com outras morbidades crônicas que, frequentemente, precisam visitar ambulatórios médicos experienciam maiores níveis de estresse pela exposição nesses serviços.

Os jovens, especialmente os grupos mais vulneráveis da população, como os trabalhadores informais e desempregados, podem ser impactados pelas medidas de contingenciamento da COVID-19 diante das incertezas sobre o trabalho. A pandemia da COVID-19 pode também afetar os pensamentos, comportamentos e respostas emocionais das crianças, sobretudo aquelas separadas de seus cuidadores durante este processo. (WHO, 2004) Dessa forma, aquelas que estão em quarentena em hospitais ou centros de atendimento e as que têm pais infectados podem ser mais suscetíveis a problemas de saúde mental, devido ao sofrimento e medo causados pela perda ou separação dos pais. (WHO, 2004)

O fechamento das escolas, a redução das atividades ao ar livre e da interação social podem explicar o aumento de sintomas depressivos entre crianças. (TSAMAKIS et al., 2020; XIE et al., 2020) Os impactos psicológicos nas crianças podem ser exacerbados pela desinformação, aumentando as incertezas e dúvidas. À medida que esses processos se concentram no sistema límbico podem eclodir em forma de pânico individual em crianças ou até 
em adultos. Esses sistemas estressores podem desestabilizar os recursos de enfrentamento de um indivíduo ou de uma comunidade inteira. (HUREMOVĆ, 2019)

Pessoas confirmadas e suspeitas da COVID-19 também podem sofrer grande pressão psicológica, pois o medo de graves consequências da doença e contágio podem gerar solidão, negação, ansiedade, depressão, insônia e desespero, e inclusive diminuir a adesão ao tratamento. (XIANG et al., 2020) Casos isolados suspeitos podem sofrer de ansiedade devido à incerteza sobre seu estado de saúde e desenvolver sintomas obsessivo-compulsivos, como repetidas verificações de temperatura e esterilização. (LI, W. et al., 2020b)

Pacientes hospitalizados por COVID-19 também enfrentam desafios que podem impactar na saúde mental, estes enfrentam o tratamento afastados da família e em situação de isolamento por tempo indefinido, o que pode gerar sentimento de solidão, desespero e ansiedade. $\mathrm{O}$ sofrimento psíquico relacionado ao medo da morte também pode ocorrer, além das possíveis sequelas que poderiam aumentar o risco de depressão caso, ao retornar para casa, estes não consigam desempenhar suas atividades anteriores. Ademais, as pessoas que foram diagnosticadas pela doença podem sofrer o estigma social.

Pacientes com problema de saúde mental anterior à pandemia podem apresentar recidivas em seu estado mental, como ataques de pânico desencadeados pelo medo. (KOZLOFF et al., 2020; TSAMAKIS et al., 2020) Esses pacientes podem enfrentar dificuldades para receber tratamento ou até para dar continuidade a tratamento iniciado antes da COVID-19 e, portanto, aumentar o risco de recaída a crises ou surtos e comportamentos incontroláveis. (LI, W. et al., 2020)

Indivíduos com esquizofrenia e transtornos relacionados podem estar em maior risco de se infectarem com COVID-19 devido à própria característica desses transtornos, como delírios, 
alucinações, comportamento desorganizado, comprometimento cognitivo e percepção insatisfatória. (KOZLOFF et al., 2020) Além disso, podem apresentar recidivas caso a disponibilidade de medicação regular ou à adesão à medicação sofram alterações durante a pandemia. (CHATTERJEE; BARIKA; MUKHERJEE, 2020) A continuidade da assistência a esses pacientes é de fundamental importância para evitar a descompensação durante a pandemia e suas posteriores consequências. (KOZLOFF et al., 2020)

Implicações psicológicas também são observadas na população em geral. (BACON; CORR, 2020; LEI et al., 2020; MOGHANIBASHI-MANSOURIEH, 2020) A preocupação com as consequências econômicas e futuras; a exposição constante às notícias da COVID-19; o aumento do risco de contrair a doença e a preocupação com o estado de saúde de familiares, amigos e colegas são alguns dos motivos que contribuem com o aumento da ansiedade. (MOGHANIBASHI-MANSOURIEH, 2020)

Alguns autores alertam para a maior ocorrência de transtornos mentais e sintomas depressivos e de ansiedade entre mulheres durante a pandemia. (DØRHEIM; BJORVATN; EBERHARD-GRAN, 2012; QIU et al., 2020) Dentre os fatores elencados como possíveis causadores estão: maior vulnerabilidade socioeconômica; maior exercício do papel de principais cuidadoras dentro de casa, inclusive de pessoas em grupos de risco; e pelo fato de estarem em maior número entre profissionais de saúde da linha de frente. (QIU et al., 2020)

Familiares de vítimas fatais da COVID-19 enfrentam desafios que podem impactar na sua saúde mental relacionados, por exemplo, a um óbito inesperado que pode ser bastante doloroso. Além disso, os desafios práticos impostos pelas características da COVID-19, como a proibição de contato com o paciente durante os últimos dias de vida e a inviabilidade de estar presente ou mesmo de realizar um enterro. As pandemias perturbam o 
senso de realidade e ordem, levando a uma maneira distinta de armazenar e metabolizar memórias e experiências. $\mathrm{O}$ retorno à normalidade pode ser acompanhado por repressão e até amnésia do caos e do trauma que precederam a recuperação. (HUREMOVIĆ, 2019) Esses familiares de vítimas da COVID-19 que vieram a óbito podem apresentar maior risco de transtorno relacionado ao luto prolongado, o que pode comprometer o seu bem-estar físico e mental. (ZHAI; DU, 2020)

\section{Transtornos mentais e sintomas associados à COVID-19}

\section{Ansiedade}

Uma revisão de literatura sobre saúde mental e COVID-19 indicou que do total de 43 artigos selecionados, 33 artigos, ou seja, 77\% sugeriam uma relação entre sintomas de ansiedade e pandemia da COVID-19. (MACHADO et al., 2020) Foram encontrados elevados escores de ansiedade associados ao diagnóstico de coronavírus, enfrentamento de álcool/drogas, desesperança extrema e ideação suicida. (LEE, 2020) E altos níveis de ansiedade entre a população, com prevalência de até $80 \%$ de preocupação relacionada à COVID-19. (ROY et al., 2020) As questões associadas ao risco de ansiedade e depressão estavam relacionadas ao medo da pandemia da COVID-19 e à fragilidade sobre as questões clínicas dos pacientes, medo de ser infectado e de adoecer (PULVIRENTI et al., 2020), e de que familiares contraíssem a COVID-19 (75,2\%). (WANG, C. et al., 2020) A ansiedade relacionada à escassez de medicamentos também foi uma das questões citadas como fontes geradoras de preocupação. (PULVIRENTI et al., 2020) Emoções negativas - ansiedade, depressão e indignação - aumentaram 
enquanto que as emoções positivas - mensuradas pelos escores de felicidade de Oxford - e satisfação com a vida diminuíram com a pandemia. (LI, S. et al., 2020)

Um estudo realizado na China encontrou maior índice de ansiedade (29\%), consumo perigoso, prejudicial ou dependência de álcool (30\%), e menor bem-estar mental do que o habitual $(32,1 \%)$ entre 1.074 pessoas da província chinesa de Hubei, que responderam ao questionário on-line da pesquisa. (AHMED et al., 2020) Outro estudo demonstrou incidências de ansiedade total, leve, moderada e grave de $18,1 \%, 10,5 \%, 5,7 \%$ e $1,9 \%$, respectivamente. Os entrevistados que tiveram experiência de exposição à COVID-19 relataram taxas mais altas de ansiedade acompanhada de depressão do que os que não tiveram experiência de exposição (taxas de incidência de 31,6\% e 12,6\%, respectivamente). (CHEN et al., 2020)

Já Lei e demais autores (2020) encontraram prevalência de $8,3 \%$ de ansiedade e $14,6 \%$ de depressão em estudo realizado na China. A prevalência foi maior no grupo de pessoas afetadas pelo distanciamento físico - a própria pessoa ou seus contatos próximos estiveram em quarentena - $(12,9 \%, 22,4 \%)$ do que no grupo não afetado $(6,7 \%, 11,9 \%)$. Menor renda média familiar, menor nível de escolaridade, maior preocupação em ser infectado pela COVID-19, não contar com apoio psicológico, maior perda econômica e piores condições de saúde autorreferida foram associados com escores mais altos de ansiedade e depressão. (LEI et al., 2020)

\section{Depressão}

A revisão de literatura, anteriormente citada, também indicou alto número de estudos empíricos investigando depressão durante a pandemia da COVID-19. Do total de 43 artigos selecionados, 56\% $(n=26)$ investigaram a relação entre COVID-19 e depressão ou sintomas depressivos. (MACHADO et al., 2020) Dentre os estudos 
citados, uma investigação realizada na China com 1.593 participantes, encontrou prevalência de depressão de aproximadamente 15\%. (LEI et al., 2020) Enquanto em áreas mais afetadas da China foi observada prevalência de $50,4 \%$ de sintomas de depressão. (WANG, C. et al., 2020) Indivíduos residentes fora da província de Hubei apresentaram menor chance de experimentar sintomas de angústia em comparação com aqueles em Wuhan (epicentro inicial). (WANG, C. et al., 2020)

Outro estudo na China encontrou prevalência de depressão e combinação de depressão e ansiedade durante o período da COVID-19 de $48,3 \%$ e 19,4\%, respectivamente. (GAO et al., 2020) No Vietnam, foi observada prevalência aumentada de depressão (29,2\%) em pacientes que apresentaram infecção por COVID-19. (NGUYEN et al., 2020) Houve aumento da prevalência de depressão comórbida com ansiedade tanto em pacientes que apresentaram infecção por COVID-19 (21,1\%) quanto no público em geral $(22,4 \%)$. Os participantes da pesquisa - 3.947 pessoas recrutadas em departamentos ambulatoriais de nove hospitais e centros de saúde que tiveram o diagnóstico de COVID-19 apresentaram maior probabilidade de depressão e menor escore na escala que mede saúde e qualidade de vida. Conhecimentos em saúde (health literacy) foi um fator de proteção para depressão e maior qualidade de vida durante a epidemia da COVID-19, especialmente entre pessoas não diagnosticadas com a doença. (NGUYEN et al., 2020)

Na Itália, um estudo com 500 indivíduos demonstrou probabilidade de sofrimento psíquico, 19,4\% leve e 18,6\% moderada a grave. (MOCCIA et al., 2020) Temperamentos ciclotímicos, depressivos e ansiosos, e "Necessidade de aprovação" foram fatores de risco para sofrimento psíquico moderado a grave em comparação com nenhum sofrimento. (MOCCIA et al., 2020)

Outro estudo na China, analisando o Transtorno de Ansiedade Generalizada (TAG), sintomas depressivos e qualidade do sono do 
público, demonstrou prevalências gerais de 35,1\%, 20,1\% e 18,2\%, respectivamente. (HUANG; ZHAO, 2020) Jovens relataram maior prevalência de TAG e sintomas depressivos do que os idosos. Profissionais de saúde foram mais propensos a ter má qualidade do sono em comparação com outros grupos ocupacionais. A idade $<35$ anos e o tempo gasto com foco na COVID-19 $\geq 3$ horas por dia foram associados ao TAG. (HUANG; ZHAO, 2020)

\section{Alterações no sono}

Alterações no sono também têm sido um dos sintomas mais frequentemente relatados nos estudos realizados durante a pandemia da COVID-19. (MACHADO et al., 2020) Huang e Zhao (2020) encontraram na China prevalência geral de má qualidade do sono em 18,2\% dos pesquisados. Essa prevalência foi maior em profissionais de saúde que passaram tempo $\geq 3$ horas/dia no atendimento a pacientes da COVID-19 comparados àqueles com $<1$ hora/dia e 1-2 horas/dia. (HUANG; ZHAO, 2020)

Roy e demais autores (2020) relataram alterações negativas do sono em $12,5 \%$ dos participantes da pesquisa, enquanto que Liu e demais autores (2020) demonstraram que participantes com melhor qualidade do sono ou menor frequência de acordar durante a noite relataram menor TEPT. No estudo de Xiao e demais autores (2020), a ansiedade foi associada ao maior estresse e à qualidade do sono reduzida, enquanto maiores níveis de capital social foi associado positivamente ao aumento da qualidade do sono. Um estudo realizado na China, incluindo um total de 1.563 participantes, demonstrou que 36,1\% dos participantes apresentaram sintomas de insônia, de acordo com o Índice de Gravidade da Insônia (IGI) (pontuação total $\geq 8$ ). (ZHANG; MA, 2020) O maior risco de insônia estava associado a: menor nível de escolaridade; trabalho atual em unidade de isolamento; preocupação em ser infectado; falta de apoio psicológico; alta exposição a notícias ou 
mídias sociais sobre a COVID-19; e forte incerteza em relação ao controle eficaz da doença. (ZHANG; MA, 2020)

\section{Comportamento obsessivo}

Fatores relacionados a comportamentos obsessivos (GONG et al., 2020; ROY et al., 2020; TIAN et al., 2020; ZHANG, W. et al., 2020) também têm sido abordados durante a pandemia da COVID-19. Dentre eles, um estudo realizado na China observou prevalência de $70 \%$ de alterações psicológicas moderadas ou altas, com pontuações especificamente elevadas para compulsão obsessiva, sensibilidade interpessoal, ansiedade fóbica e psicoticismo. (TIAN et al., 2020) Aqueles com mais de 50 anos, com melhor nível educacional, divorciados ou viúvos, e que exerciam trabalho agrícola apresentaram mais sintomas. Os mais jovens e a equipe médica estavam no grupo de mais alto risco em termos da gravidade dos sintomas psicológicos. (TIAN et al., 2020) Outro estudo também realizado na China encontrou que morar em áreas rurais, ser mulher e estar em risco de contato com pacientes diagnosticados com COVID-19 foram os fatores de risco mais comuns para obsessivos-compulsivos. (ZHANG, W. et al., 2020) Também na China, médicos apresentaram maior prevalência de sintomas obsessivo-compulsivos em comparação a outros profissionais de saúde e apresentaram escores totais mais altos de sintomas obsessivos-compulsivos no teste psicológico Generalized Anxiety Disorder 2-item (GAD-2) e Symptom Checklist-90-Revised (SCL-90-R). (ZHANG, W. et al., 2020)

No Vietnam, a epidemia de COVID-19 acarretou em pânico e hipocondria, induzindo a comportamentos de busca desnecessária a cuidados em saúde e aumento da demanda a serviços de assistência médica entre as pessoas consultadas on-line entre 
a população em geral. (GONG et al., 2020) Já na Índia, a preocupação excessiva com o uso de luvas e desinfetantes, comportamento que pode aumentar os sintomas obsessivos, foi citada por $72 \%$ dos participantes de uma pesquisa. (ROY et al., 2020) Os participantes relataram também sintomas como alterações negativas do sono (12,5\%), paranoia sobre a utilização de mídias sociais relacionadas à infecção por COVID-19 (37,8\%) e angústia (36,4\%). (ROY et al., 2020).

\section{Transtorno de Estresse Pós-Traumático (TEPT)}

Além de sintomas de ansiedade, depressão e alterações no sono, o TEPT tem sido relacionado à COVID-19. (MACHADO et al., 2020) Liu e demais autores (2020) encontraram 7\% de prevalência de sintomas de TEPT nas áreas mais atingidas pelo surto de COVID-19 na China. As mulheres tiveram TEPT mais alto, apresentando alterações negativas na cognição, humor ou hiperexcitação. Participantes com melhor qualidade do sono ou menor frequência de acordar durante a noite relataram menor TEPT. (LIU et al., 2020)

Tan e demais (2020) encontraram que 10,8\% dos respondentes atendiam ao diagnóstico de TEPT após retornar ao trabalho. Chew e demais autores (2020) verificaram alto risco de TEPT (OR 2,20; IC95\% 1,12-4,35) associado à presença de sintomas físicos experimentados no mês anterior entre profissionais de saúde. Um grande número de participantes relatou mais de quatro sintomas $(33,4 \%)$, dentre esses, dor de cabeça foi o sintoma mais comumente relatado $(32,3 \%)$. 


\section{Estratégias para a atenção à saúde mental}

Os estudos até então publicados apontam para a necessidade de inclusão de cuidados de saúde mental enquanto a pandemia ainda está em curso, para evitar maiores problemas futuros e possivelmente reduzir a duração e o custo do tratamento dos efeitos psicológicos posteriores. Esses estudos alertam também para medidas específicas para os grupos mais vulneráveis da população ou grupos que podem ser mais afetados pela COVID-19.

\section{Estratégias para grupos específicos}

As discussões dos estudos indicaram a importância do apoio aos profissionais de saúde, especialmente os da linha de frente de combate à pandemia, por meio de intervenções em saúde mental. (KANG et al., 2020) Destacam-se os trabalhadores alocados nos departamentos de doenças respiratórias, de emergência, Unidade de terapia intensiva (UTI) e doenças infecciosas. (LI, Z. et al., 2020; LU et al., 2020)

As principais medidas citadas foram: aumentar a disponibilidade de tratamento especializado com psicólogos e psiquiatras (KANG et al., 2020; TIAN et al., 2020); viabilizar intervenções multidisciplinares, abordando as manifestações psicológicas e os sintomas físicos, aconselhamentos dedicados a aliviar o medo de transmitir a infecção aos membros da família e aumentar a confiança e a autoestima (CHEW et al., 2020); fortalecer o apoio de colegas no local de trabalho, desenvolvimento de fóruns on-line ou linhas diretas de aconselhamento e identificação precoce dos fatores de risco. (TAN et al., 2020; XU et al., 2020)

Além disso, os profissionais de saúde precisam de condições de trabalho adequadas, com provisão de equipamento médico 
de proteção suficiente, tempo de descanso adequado, bem como o acesso a programas para aumento da capacidade de resiliência e bem-estar psicológico. (ZHANG, W. et al., 2020) É importante mobilizá-los à busca ativa por seus sistemas de apoio social. Atividades de lazer e treinamento sobre como relaxar devem ser organizadas para ajudar a equipe a reduzir o estresse. (MO et al., 2020)

Já entre os pacientes sob cuidados clínico-domiciliares, destaca-se a importância de realização de uma avaliação periódica da qualidade de vida. É necessário cuidar de pacientes em risco, planejando um suporte médico e psicológico individualizado ao longo da vida, sobretudo em casos excepcionais, como na pandemia por COVID-19. (PULVIRENTI et al., 2020)

Já as crianças demandam uma detecção precoce de possíveis problemas de saúde mental. O fornecimento oportuno de intervenções psicológicas ajudará os pais a cuidar melhor de seus filhos enquanto no hospital e ajudará as crianças na recuperação e alta pós-tratamento. (YUAN et al., 2020) É também necessária especial atenção à saúde psicológica de indivíduos menores de 18 anos e acima de 50 anos, disponibilizando intervenções psicológicas por meio de canais de televisão, internet e telefone, com orientações para o entendimento da epidemia e para aliviar o pânico e o medo. (TIAN et al., 2020)

\section{Políticas públicas e população}

Em relação a políticas públicas, os governos devem primeiramente reconhecer a COVID-19 como uma preocupação emergencial de saúde pública para poder melhorar o conhecimento em saúde (health literacy) e controlar a doença e suas consequências ainda durante o surto. (NGUYEN et al., 2020) Para o público, é de fundamental 
importância que as informações e conhecimentos sobre a pandemia sejam transparentes, atualizados, precisos, breves e simples para poder ajudar a controlar melhor a doença. (HUANG; ZHAO, 2020; NGUYEN et al., 2020) Recomenda-se o desenvolvimento de uma plataforma oficial, integrada e uniforme em saúde mental para fornecer aconselhamento psicológico às pessoas necessitadas. (HUANG; ZHAO, 2020) A identificação oportuna de sofrimento psíquico e a classificação precisa das necessidades de saúde mental entre as populações mais afetadas facilitarão o desenvolvimento de intervenções psicológicas direcionadas. (ZHANG, J. et al., 2020)

É necessário formular intervenções psicológicas para melhorar a saúde mental e resiliência durante a epidemia da COVID-19. (WANG, C. et al., 2020) Governos devem adotar políticas de subsídios apropriadas para aliviar a pressão econômica causada pela epidemia na população (TIAN et al., 2020), aumentar o apoio médico (LEI et al., 2020), além de implementar políticas públicas que estimulem o capital social durante o isolamento. (XIAO et al., 2020) $\mathrm{O}$ apoio social não apenas reduz a pressão psicológica durante as epidemias, mas também muda a atitude em relação a métodos de busca de ajuda. (CAO et al., 2020)

\section{Reorganização dos serviços psicossociais no Brasil}

A Política Nacional de Saúde Mental do Brasil compreende estratégias e diretrizes adotadas para organizar a assistência às pessoas com necessidades de tratamento e cuidados específicos em saúde mental. (BRASIL, 2001) A partir de 2011, com a Portaria $\mathrm{n}^{\circ} 3.088 / 2011$, os serviços de saúde mental passaram a se organizar seguindo a lógica das Redes de Atenção à Saúde (RAS) - sistema integrado que opera de forma contínua e voltado para as 
condições agudas e crônicas de saúde/doença, estabelecendo a rede temática e prioritária de cuidados em saúde mental: Rede de Atenção Psicossocial (RAPS). (BRASIL, 2011)

$\mathrm{O}$ acolhimento das pessoas com necessidade de tratamento em saúde mental e de seus familiares é uma estratégia de atenção fundamental para o alívio do sofrimento, para a identificação das necessidades assistenciais e planejamento de intervenções medicamentosas e terapêuticas. Os indivíduos em situações de crise podem ser atendidos em qualquer serviço da RAPS, formada por várias unidades com finalidades distintas, de forma integral e gratuita, pela rede pública de saúde. (MENDES, 2011)

O Ministério da Saúde (MS), através da Nota Técnica $n^{\circ}$ 12/2020, reconheceu a importância de se adotar medidas de prevenção, controle e contenção de riscos, danos e agravos à saúde pública no âmbito da atenção psicossocial, durante as ações de controle da pandemia. Além do apoio às medidas de mitigação do seu avanço, há recomendações para o seguimento das ações da RAPS, durante o período de distanciamento físico, através de protocolos. Assim, é recomendada a manutenção das atividades assistenciais, com suporte às pessoas em situação de crise, manejo das agudizações e exacerbações, riscos e seguimento terapêutico. Os serviços que compõem a rede, como os Centros de Atenção Psicossocial (CAPS), hospitais especializados e as residências terapêuticas, precisam manter o funcionamento durante a pandemia. Neste momento, os atendimentos dos serviços de saúde mental requerem atenção sobre o manejo, distinção de reações esperadas e indicadores de risco, como sintomas persistentes, comprometimento significativo do funcionamento social e cotidiano, ou complicações associadas aos transtornos preexistentes ou à situação de pandemia, como as ideações e as tentativas de suicídio, que requerem uma atenção especializada. Em relação à farmacoterapia, ao passo que é importante manter a continuidade das medicações em uso, é necessária 
atenção à distribuição de maior quantidade. Essa medida pode também contribuir para diminuir os contatos com os serviços de saúde, mas também pode contribuir para tentativas de suicídio ou provocar intoxicações.

Alguns critérios podem ser observados para identificar se uma reação está se tornando sintomática e precisa de encaminhamento para a RAPS, como dificuldades profundas na vida familiar, social ou no trabalho, risco de complicações, em especial risco de suicídio. Além de problemas coexistentes como alcoolismo ou outras dependências, depressão maior, psicose e transtorno de estresse pós-traumático e padrões de sofrimento prolongado como tristeza, medo generalizado e ansiedade expressos corporalmente, sintomas que podem vir a desencadear uma patologia a médio ou longo prazo, caso não seja realizada uma intervenção qualificada. (BRASIL, 2020b)

Consonante com a Nota Técnica $\mathrm{n}^{\circ} 12 / 2020$, os municípios e estados também têm divulgado os Planos de Contingência de Saúde Mental e outras diretrizes para o funcionamento dos serviços neste período. (BRASIL, 2020a) A manutenção das ações da RAPS, como matriciamento pelas equipes de saúde mental e equipe da Atenção Primária em Saúde (APS) por meios on-line, a manutenção dos acolhimentos de novos casos e dos atendimentos emergenciais, mantendo a renovação de receitas e a dispensa de medicamentos têm sido enfatizadas como ações prioritárias. Em contrapartida, tem sido recomendada a suspensão das atividades em grupos e consultas não emergenciais, além de cautela e análise caso a caso das visitas domiciliares, especialmente a idosos e outros grupos de risco. 


\section{Considerações finais}

A vida da maioria das pessoas será afetada de alguma forma pela pandemia da COVID-19 nos próximos meses e, talvez, nos próximos anos, principalmente os grupos mais vulneráveis da população. É importante o desenvolvimento e manutenção de ações de atenção à saúde mental em conjunto com as ações de controle da pandemia. Os desfechos em saúde mental mais abordados pelos estudos empíricos, até então publicados, foram ansiedade, depressão ou sintomas depressivos, comportamentos obsessivos, Transtorno Obsessivo-Compulsivo (TOC), trauma ou transtorno de estresse pós-traumático, sofrimento psicológico, estresse e medo.

No entanto, a maioria dos estudos apresentava significativos limites metodológicos. Investimentos em novas pesquisas, com estudos controlados ou seguimento longitudinal, são necessários para maior exploração das relações existentes entre a pandemia da COVID-19 e a saúde mental das pessoas. Enquanto esses estudos não são realizados, considerando os efeitos das epidemias anteriores sobre a saúde mental (durante e após), medidas governamentais precisam ser implementadas para reduzir os potenciais efeitos catastróficos da atual pandemia da COVID-19 e o ônus que permanecerá pós-pandemia.

São necessárias novas estratégias para garantir a continuidade do acesso a medicamentos psiquiátricos e à psicoterapia, intervenções psicológicas direcionadas, além da organização dos serviços e sistemas de saúde, durante a pandemia, utilização de ferramentas on-line, quando possível e incentivos à socialização à longa distância.

No Brasil, para a redução do sofrimento psicológico e prevenção de novos problemas de saúde mental durante o contingenciamento da COVID-19, recomenda-se: 
1. Maior atenção aos grupos mais vulneráveis, como os trabalhadores de saúde, especialmente os de linha de frente, às pessoas idosas, às mulheres, às pessoas com menor acesso aos serviços de saúde e aos pacientes com doença crônica preexistente que necessitam manter a frequência de utilização dos serviços de saúde;

2. Melhoria do acesso à RAPS, através de formas de atendimento on-line e atualização da disposição de informações sobre o funcionamento dos serviços de forma fácil, segura e rápida para que a população conheça os serviços e possam acessá-los;

3. Planejamento estratégico nacional e coordenação de primeiros socorros por teleatendimentos;

4. Coordenação da RAPS para crises mais abrangentes, incluindo monitoramento epidemiológico, triagem, encaminhamento e intervenção direcionada, além da manutenção dos serviços de acolhimento de novos casos e acompanhamento dos usuários de serviços de saúde mental.

\section{Referências}

AHMED, M. D. Z. et al. Epidemic of COVID-19 in China and associated Psychological Problems. Asian Journal of Psychiatry, Amsterdam, v. 51, p. 102092, 2020.

AQUINO, E. M. L. et al. Medidas de distanciamento social no controle da pandemia de COVID-19: potenciais impactos e desafios no Brasil. Ciência \& Saúde Coletiva, Rio de Janeiro, v. 25, supl. 1, p.2423-2446, 2020.

ASMUNDSON, G. J. G. et al. Health anxiety: Current perspectives and future directions. Current Psychiatry Reports, Philadelphia, v. 12, n. 4, p. 306-312, 2010. 
BACON, A. M.; CORR, P. J. Coronavirus (COVID-19) in the United Kingdom: a personality-based perspective on concerns and intention to self-isolate. British Journal of Health Psychology, Leicester, v. 25, n. 3, p. 1-10, Sept. 2020.

BAO, Y. et al. 2019-nCoV epidemic: address mental health care to empower society. The Lancet, London, v. 395, n. 10224, p. e37-e38, 2020.

BECK, J. S. Terapia cognitivo-comportamental: teoria e prática. Porto Alegre: Artmed, 2013.

BOLETIM COVIDA: pandemia de COVID-19: os impactos das medidas de distanciamento social e redução de fluxo intermunicipal na Bahia. [Salvador]: Rede Covida, n. 2, 11 abr. 2020. Disponível em: https://redecovida.org/mainsite-covida/wp-content/uploads/2020/04/BoletimCovida_2ed_Final.pdf. Acesso em: 22 nov. 2020.

BRASIL. Lei $n^{\circ} 10.216$, de 6 de abril de 2001. Dispõe sobre a proteção e os direitos das pessoas portadoras de transtornos mentais e redireciona o modelo assistencial em saúde mental. Diário Oficial da União, Brasília, DF, 9 abr. 2001.

BRASIL. Ministério da Saúde. Portaria n³.088, de dezembro de 2011. Institui a Rede de Atenção Psicossocial para pessoas com sofrimento ou transtorno mental e com necessidades decorrentes do uso de crack, álcool e outras drogas, no âmbito do Sistema Único de Saúde (SUS). Diário Oficial da União, Brasília, DF, 2011.

BRASIL. Ministério da Saúde. Secretaria de Atenção Primária. Nota Técnica n 12/2020. Recomendações à Rede de Atenção Psicossocial sobre estratégias de organização no contexto da infecção da Covid-19 causada pelo novo coronavirus (SARS-CoV-2). Brasília, DF: Ministério da Saúde, Secretaria de Atenção Primária, 2020.

BRASIL. Saúde Mental e Atenção Psicossocial na Pandemia COVID-19. Brasília, DF: Ministério da Saúde: Fundação Oswaldo Cruz, 2020b.

CAO, W. et al. The psychological impact of the COVID-19 epidemic on college students in China. Psychiatry Research, Amsterdam, v. 7, n. 1, p. 105-110, 2020.

CHATTERJEE, S. S.; BARIKAR C, M.; MUKHERJEE, A. Impact of COVID-19 pandemic on pre-existing mental health problems. Asian Journal of Psychiatry, Amsterdam, v. 51, p. $102071,2020$.

$\mathrm{CHEN}$, Y. et al. Prevalence of self-reported depression and anxiety among pediatric medical staff members during the COVID-19 outbreak in Guiyang, China. Psychiatry Research, Amsterdam, v. 288, p. 113005, 2020. 
CHEW, N. W. S. et al. A multinational, multicentre study on the psychological outcomes and associated physical symptoms amongst healthcare workers during COVID-19 outbreak. Brain Behavior and Immunity, San Diego, v. 88, p. $559-565.2020$.

DØRHEIM, S. K.; BJORVATN, B.; EBERHARD-GRAN, M. Insomnia and Depressive Symptoms in Late Pregnancy: A Population-Based Study. Behavioral Sleep Medicine, London, v. 10, n. 3, p. 152-166, 2012.

GAO, J. et al. Mental health problems and social media exposure during COVID-19 outbreak. PLoS ONE, San Francisco, v. 15, n. 4, p. 1-10, Feb. 2020.

GONG, K. et al. Internet Hospitals Help Prevent and Control the Epidemic of COVID-19 in China: Multicenter User Profiling Study. Journal of Medical Internet Research, Toronto, v. 22, n. 4, 2020.

HUANG, Y.; ZHAO, N. Generalized anxiety disorder, depressive symptoms and sleep quality during COVID-19 outbreak in China: a web-based cross-sectional survey. Psychiatry Research, Amsterdam, v. 288, p. 112954, 2020.

HUREMOVIĆ, D. Psychiatry of pandemics: a mental health response to infection outbreak. Chan: Springer, 2019.

KANG, L. et al. Impact on mental health and perceptions of psychological care among medical and nursing sta ff in Wuhan during the 2019 novel coronavirus disease outbreak: a cross-sectional study. Brain, Behavior, and Immunity, San Diego, v. 87, p. 11-17., 2020.

KOZLOFF, N. et al. OUP accepted manuscript. Schizophrenia Bulletin, [s. l.] v. 46, n. 4, p. 752-757, July 2020.

LEE, S. A. Coronavirus anxiety scale: A brief mental health screener for COVID-19 related anxiety. Death Studies, Washington, v. 44, n. 7, p. 1-9, 2020.

LEI, L. et al. Comparison of Prevalence and Associated Factors of Anxiety and Depression Among People Affected by versus People Unaffected by Quarantine During the COVID-19 Epidemic in Southwestern China. Medical Science Monitor, Smithtown, 2020.

LI, S. et al. The impact of COVID-19 epidemic declaration on psychological consequences: a study on active weibo users. International Journal of Environment and Public Health, Basel, v. 17, n. 6, p. 2032, 2020. 
LI, W. et al. Progression of Mental Health Services during the COVID-19 Outbreak in China. International Journal of Biological Sciences, Lake Haven, v. 16, n. 10, p. 1732-1738, 2020.

$\mathrm{LI}$, Z. et al. Vicarious traumatization in the general public, members, and nonmembers of medical teams aiding in COVID-19 control. Brain, Behavior, and Immunity, San Diego, v. 88, p. 916-919, 2020.

LIU, N. et al. Prevalence and predictors of PTSS during COVID-19 Outbreak in China Hardest-hit Areas: Gender differences matter. Psychiatry Research, Amsterdam v. 287, p. 112921, 2020.

LU, W. et al. Psychological status of medical workforce during the COVID-19 pandemic: A cross-sectional study. Psychiatry Research, Amsterdam, v. 288, p. 1-5, 2020.

LUPIEN, S. J. et al. Effects of stress throughout the lifespan on the brain, behaviour and cognition. Nature Reviews Neuroscience, London, v. 10, n. 6, p. 434-445, 2009. MACHADO, D. B. et al. Effects of COVID-19 on Anxiety, Depression and Other Mental Health Issues: A worldwide scope. Research Square, [s. l.], 2020.

MENDES, E. V. As redes de atenção à saúde. Brasília, DF: Organização Pan- Americana da Saúde, 2011.

MO, Y. et al. Work stress among Chinese nurses to support Wuhan for fighting against the epidemic COVID-19. Journal of Nursing Management, Oxford, v. 28, n. 5, p. 1002-1009, 2020.

MOCCIA, L. et al. Affective temperament, attachment style, and the psychological impact of the COVID-19 outbreak: an early report on the Italian general population. Brain Behavior and Immunity, San Diego, v. 87, p. 75-79, 2020.

MOGHANIBASHI-MANSOURIEH, A. Assessing the anxiety level of Iranian general population during COVID-19 outbreak. Asian Journal of Psychiatry, Amsterdam, v. 51, p. 102076, 2020.

NGUYEN, H. C. et al. People with Suspected COVID-19 Symptoms Were More Likely Depressed and Had Lower Health-Related Quality of Life: The Potential Benefit of Health Literacy. Journal of Clinical Medicine, Basel, v. 9, n. 4, p. 965, 2020.

ORGANIZAÇÃO MUNDIAL DA SAÚDE. CID-10: classificação estatística internacional de doenças. São Paulo: EdUSP, 1994. v. 1 
PULVIRENTI, F. et al. Health-related quality of life in common variable immunodeficiency Italian patients switched to remote assistance during the COVID-19 pandemic. The Journal of Allergy and Clinical Immunology in Practice, New York, v. 8, n. 6, p. 1894-1899.e2, 2020.

PURGATO, M. et al. Psychological therapies for the treatment of mental disorders in low- and middle-income countries affected by humanitarian crises. Cochrane Database of Systematic Reviews, Oxford, v. 7, n. 7, 2018.

QIU, H. et al. Clinical and epidemiological features of 36 children with coronavirus disease 2019 (COVID-19) in Zhejiang, China: an observational cohort study. The Lancet Infectious Diseases, London, v. 20, n. 6, p. 1-8, 2020.

ROY, D. et al. Study of knowledge, attitude, anxiety \& perceived mental healthcare need in Indian population during COVID-19 pandemic. Asian Journal of Psychiatry, Amsterdam, v. 51, p. 102083, 2020.

TAN, W. et al. Is Returning to Work during the COVID-19 Pandemic Stressful? A Study on Immediate Mental Health Status and Psychoneuroimmunity Prevention Measures of Chinese Workforce. Brain Behavior and Immunity, v. 87, p. 84-92, July 2020.

TIAN, F. et al. Psychological Symptoms of Ordinary Chinese Citizens Based on SCL-90 during the Level I Emergency Response to COVID-19. Psychiatry Research, Amsterdam, v. 288, p. 112992, 2020.

TORALES, J. et al. The outbreak of COVID-19 coronavirus and its impact on global mental health. International Journal of Social Psychiatry, London, v. 66, n. 4, p. 317-320. 2020.

TSAMAKIS, K. et al. COVID-19 pandemic and its impact on mental health of healthcare professionals. Experimental and Therapeutic Medicine, Athens, v. 19, n. 6, p. 3451-3453, 2020.

WANG, C. et al. Immediate Psychological Responses and Associated Factors during the Initial Stage of the 2019 Coronavirus Disease (COVID-19) Epidemic among the General Population in China. International Journal of Environment and Public Health, Basel, v. 17, n. 5, p. 1729, 2020.

WANG, Y. et al. Psychological assistance during the coronavirus disease 2019 outbreak in China. Journal of Health Psychology, London, v. 25, n. 6, p. 733-737, 2020. 
WORLD HEALTH ORGANIZATION - WHO. Investing in Mental Health. Department of Mental Health and Substance Dependence, Noncommunicable Diseases and Mental Health. Geneva: WHO, 2003.

WORLD HEALTH ORGANIZATION - WHO. The importance of caregiver-child interactions for the survival and healthy development of young children. Geneva: WHO, 2004.

XIANG, Y. T. et al. Timely mental health care for the 2019 novel coronavirus outbreak is urgently needed. The Lancet Psychiatry, Kidlington,v. 7, n. 3, p. 228-229, 2020.

$\mathrm{XIAO}, \mathrm{H}$. et al. Social capital and sleep quality in individuals who self-isolated for 14 days during the coronavirus disease 2019 (COVID-19) outbreak in January 2020 in China. Medical Science Monitor, Warsaw, v. 6, p. 1-8, 2020.

$\mathrm{XIE}, \mathrm{X}$. et al. Mental Health Status Among Children in Home Confinement During the Coronavirus Disease 2019 Outbreak in Hubei Province, China. JAMA Pediatrics, Chicago, v. 174, n. 9, p. 898-900, 2020.

$\mathrm{XU}$, J. et al. Systematic comparison of two animal-to-human transmitted human coronaviruses: SARS-CoV-2 and SARS-CoV. Viruses, Basel, v. 12, n. 2, p. 244. 2020.

YANG, Y. et al. Mental health services for older adults in China during the COVID-19 outbreak. The Lancet Psychiatry, Kidlington, v. 7, n. 4, p. e19, 2020.

YUAN, R. et al. Psychological status of parents of hospitalized children during the COVID-19 epidemic in China. Psychiatry Research, Amsterdam, n. 288, 2020.

ZHAI, Y.; DU, X. Loss and Grief amidst COVID-19: A Path to Adaptation and Resilience. Brain, Behavior, and Immunity, San Diego, v. 87, p. 80-81, 2020.

ZHANG, J. et al. The differential psychological distress of populations affected by the COVID-19 pandemic. Brain, Behavior, and Immunity, San Diego, v. 87, p. 49-50, 2020.

ZHANG, W. et al. Mental Health and Psychosocial Problems of Medical Health Workers during the COVID-19 Epidemic in China. Psychotherapy and Psychosomatics, Basel, v. 89, n. 4, p. 242-250, 2020.

ZHANG, Y.; MA, Z. F. Impact of the COVID-19 Pandemic on Mental Health and Quality of Life among Local Residents in Liaoning Province, China: A Cross-Sectional Study. International Journal of Environmental Research and Public Health, v. 17, n. 7, p. 1-12, 2020. 
